\title{
Preliminary Report of Instrumentation in Tuberculous Lumbosacral Spine
}

\author{
T Zin-Naing, MRCS \\ Department of Orthopaedic Surgery, Yangon Orthopaedic Hospital, Yangon, Myanmar
}

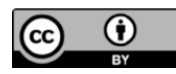

\begin{abstract}
The aims of spinal tuberculosis treatment are to eradicate the disease, to prevent the development of paraplegia and kyphotic deformity, to manage the existing deformity and neurological deficit, to allow early ambulation and to return the patient back to daily life. Methods for the treatment of tuberculosis of vertebra are still controversial. Conservative treatment includes medical therapy as well as external supports and surgery is indicated for deformity of spine, severe pain, or neurological compromise conditions. Most cases in our country were late presentations with disc space already infected, and after débridement there was a large gap needing bone graft to enhance bony fusion and anterior column support. Although the spine was infected, instrumentation posed no additional hazard in terms of tuberculous discitis. Oga et al. reported that M. tuberculosis has low adhesion capability and forms only a few microcolonies surrounded by a biofilm. Moon et al. stated that interbody fusion performed with classical anterior radical surgery per se was ineffective in the correction of kyphosis and did not prevent the increase in kyphosis angle. The present study focuses on collected clinical and radiographic outcomes in ten patients who underwent Posterior Lumbar Interbody Fusion (PLIF) for tuberculous lumbosacral spine. All the cases had instability with kyphotic deformity or loss of lordosis. Clinical outcomes were measured by Visual Analogue Scale (VAS), modified MacNab Criteria, and radiographic outcomes (segmental kyphotic angle and total lumbar lordotic, TLL, angle) on follow-up to six months. The mean VAS back scores showed decrease, and kyphotic angles and lordotic angles improved. Three cases had excellent results, six good and one fair using the modified MacNab criteria.
\end{abstract}

Key Words:

Spinal tuberculosis, Posterior Lumbar Interbody Fusion, Visual Analogue Scale, MacNab criteria

\section{INTRODUCTION}

The treatment of tuberculosis of the spine (Pott's disease) is essentially conservative and a vast majority of patients can be successfully managed on anti-tuberculous therapy (ATT) alone ${ }^{1}$. Surgery is indicated in a few specific cases where deformity of spine, pain, or neurological compromise become a serious issue. The most important route of dissemination of TB to the spine is haematogenous ${ }^{2}$. Spinal TB usually develops insidiously, and there is thus a gap of several months between onset of symptoms and appropriate medical attention.

Spinal TB patients present with various signs and symptoms which may include back or leg pain, kyphotic deformity, palpable mass in the paraspinal region, and neurological compromise, The two most important sequelae of TB spine are kyphotic deformity and neurological compromise. Neurological deficits occur due to the kyphotic deformity, spinal abscess and/or granulation tissue compressing the spinal cord or cauda equina. Most cases in our country presented late, and the disc space was already infected After débridement there was large defect which needed bone rafting to enhance bony fusion and anterior column support.

Those patients whose neurological deficit was progressing despite adequate ATT would benefit from anterolateral spinal cord decompression and instrumentation. In these patients, the histological diagnosis also needed to be established beyond doubt. Kyphotic deformity, either progressive or established, is a major indication for surgical intervention ${ }^{1}$. Furthermore, kyphotic deformity, particularly with pseudoarthrosis, can be a source of significant pain in the patient.

Kyphosis is however unusual in lumbar spine where there is a tendency for telescoping of collapsed vertebral bodies. Although the spine is infected, instrumentation poses no additional hazard in terms of tuberculous discitis. In selected patients, early operative treatment with instrumentation, when indicated, combined with chemotherapy, minimizes neurological deterioration and spinal deformity. Intractable pain is mainly due to presence of epidural mass compressing the theca and nerve roots, along with spinal instability. Both can be dealt with effectively by debridement, decompression and spinal instrumentation ${ }^{3}$. 
Table I: Demographic data of patients (source from spine registry ; YOH)

\begin{tabular}{|lccccccc|}
\hline No. & Age & Sex & Reg;No. & Operation Date & Level & Bone Graft (Bg) & $\begin{array}{c}\text { Titanium } \\
\text { Mesh+Bg }\end{array}$ \\
\hline 1 & 23 & F & 1906 & 30.5 .13 & L3,4 & + & + \\
2 & 47 & F & 1645 & 13.5 .13 & L5,S1 & + & \\
3 & 24 & M & 1088 & 25.5 .13 & L2,3 & + & + \\
4 & 53 & M & 2139 & 17.6 .13 & L3,4 & + & + \\
5 & 42 & M & 2245 & 24.6 .13 & L4,5 & + & + \\
6 & 54 & F & 2431 & 8.7 .13 & L4,5 & + \\
7 & 37 & M & 3955 & 28.10 .13 & L2,3 & + & + \\
8 & 29 & M & 4291 & 21.11 .13 & L2,3 & & + \\
9 & 33 & F & 4798 & 26.12 .13 & L4,5 & + \\
10 & 19 & F & 1098 & 3.114 & L2,3 & + \\
\hline
\end{tabular}

\section{MATERIALS AND METHODS}

This is a prospective study of clinical and radiographic outcomes in ten patients who underwent Posterior Lumbar Interbody Fusion (PLIF) for tuberculous spine. All cases operated between June 2013 and Jan 2014 were assessed with radiography, MRI and laboratory parameters before operation. All the cases had instability with kyphotic deformity or loss of lordosis. Clinical outcomes measured included Visual Analogue Scale (VAS). modified MacNab criteria at six months, neurological status and radiographic outcomes (segmental kyphotic angle and total lumbar lordotic, TLL, angle) only up to six months. To continue follow-up on cases in this sstudy, cases will be continued follow-up to 12 months and finally check with CT imaging for status of fusion,

Patients were positioned in prone position for posterior exposure. Bilateral pedicle screws were inserted at one upper and lower segment from the lesion level, and another single screw was inserted at one affected level. Rods were placed without correcting the existing kyphosis for temporary fixation. Abscess was drained after removing the lamina and infected single facet joint. Sequestered bone fragments and infected disc were removed until good cancellous bone was seen, the rods were removed and contoured to correct the kyphotic deformity, a titanium mesh filled with cancellous chip allograft 0 in affected site. With an appropriate contour obtained, rods were placed and locked. Patients were mobilized with thoracolumbar extension brace up to 12 weeks after surgery.

\section{RESULTS}

The affected vertebrae in four cases were at L2/3 level, two at L3/4, three at L4/5 and one at L5/S1. There were equal number of male and female patients, with average age of 36.1year (range, 19-54 years) (Table I). Mean operative time was 129.4 minutes. All cases were transfused with only one unit of blood during surgery; we could not assess exact amount of blood loss.
Initial pain scores in patient-reported questionnaires (VAS back) showed fast and lasting pain relief and improvement in daily activities. Mean VAS back scores decreased from 6.7 to 2.85 at three week' follow-up. After three months, mean VAS back scores decreased to 1.24, and after six months the mean VAS back scores decreased to 0.5 . Mean segmental kyphotic angle was reduced from 23.5 to 0 degree after operations, it was maintained up to six months and mean total lumbar lordotic angle improved from 23.9 to 41.5 . At three months, the mean value was 40 with no change at six months. These coronal plane angles did not change much up to 6 months follow up but we need long term follow up to further comment on this study, in addition to establish the status of fusion in all the cases.

There were no intra-operative complications. The bleeding during operation was much more in obese patients though not measured, 1 case had post-operative superficial infection but no organism was cultured and and it healed well by 6 weeks after wound débridement and dressing. Neurological examinations revealed numbness at L5 dermatome in two patients and one with one patient numbness at $\mathrm{S} 1$ dermatome area. All recovered at three months.

Modified MacNab criteria was used to evaluate the clinical results after six months of surgery. Overall, three cases had excellent results six good and one fair.

\section{DISCUSSION}

The goals of spinal tuberculosis treatment are to eradicate the disease, to prevent the development of paraplegia and kyphotic deformity, to manage the existing deformity and neurological deficit, to allow early ambulation and to return the patient back to daily life $9,11,13,19,20,23$. Methods for the treatment of tuberculosis of vertebra are still controversial. Chemotherapy is a very effective way of controlling and treating the disease and is an indispensable treatment strategy ${ }^{9,20}$. Conservative treatment includes medical therapy as well as external supports such as bed rest and/or full body plaster immobilization or body braces. Conservative management is indicated for mild to moderate form of the 

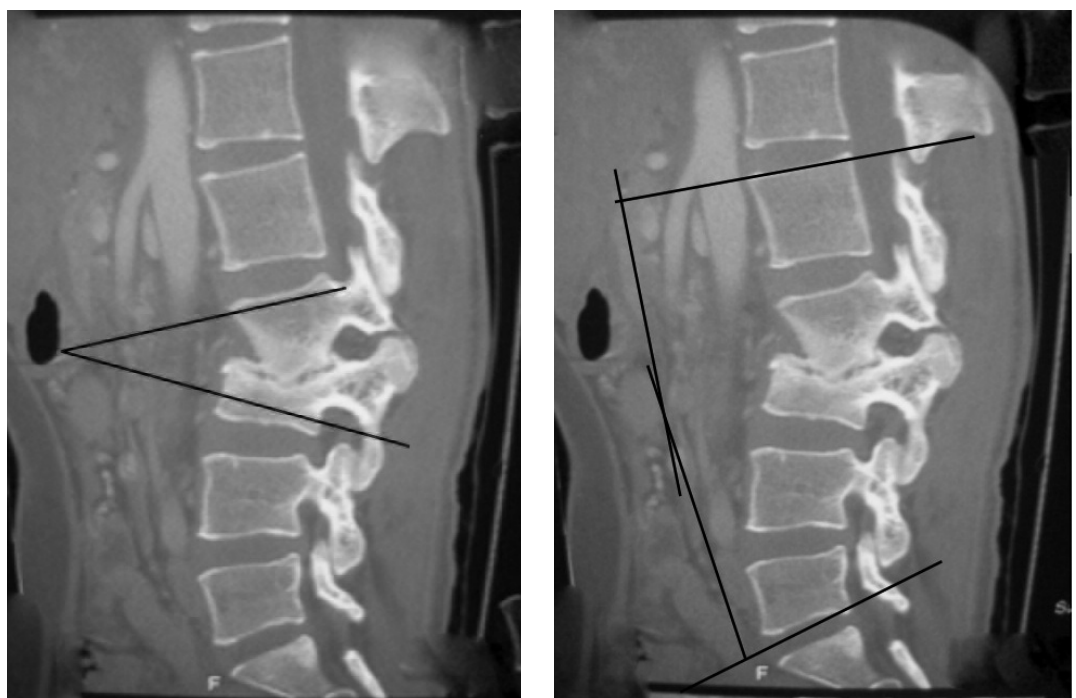

Fig. 1: Radiograph showing kyphotic angle and total lumbar lordotic angle measurements
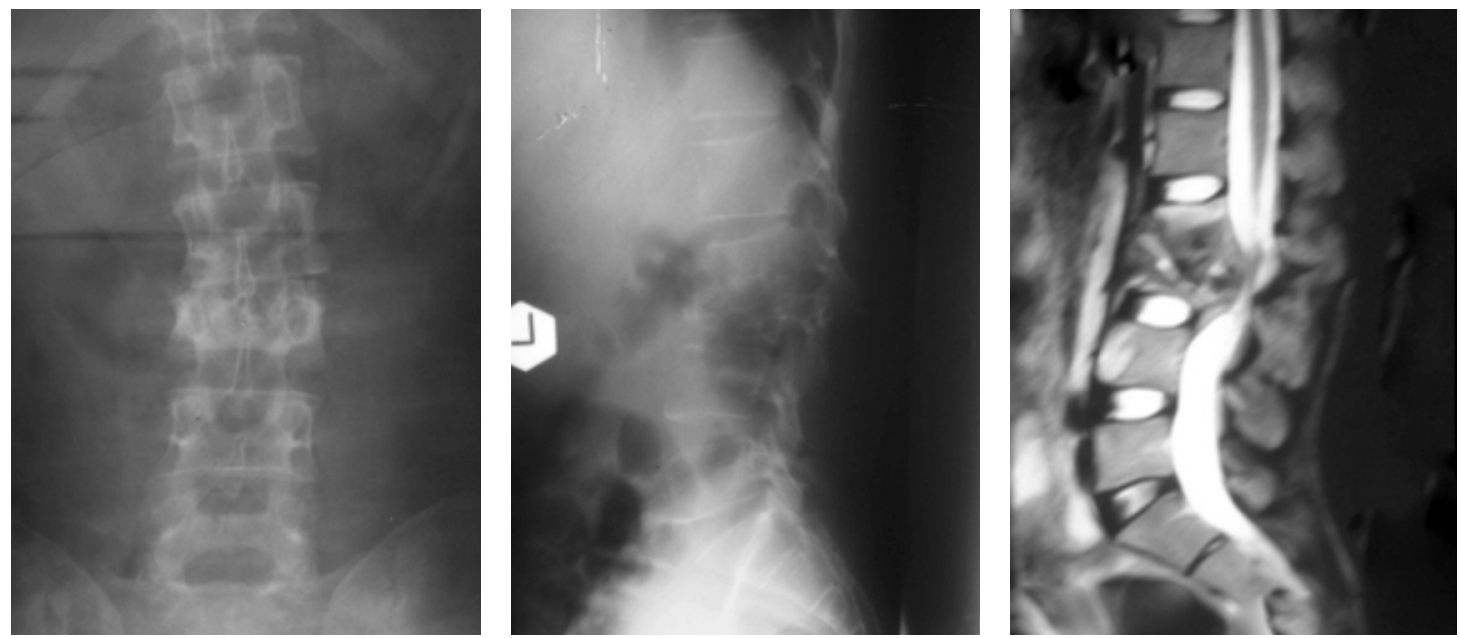

Fig. 2: Radaiographs and MRI of patient with tuberculous infection of spine L2/3 (kyphotic angle 20, TLL angle23).
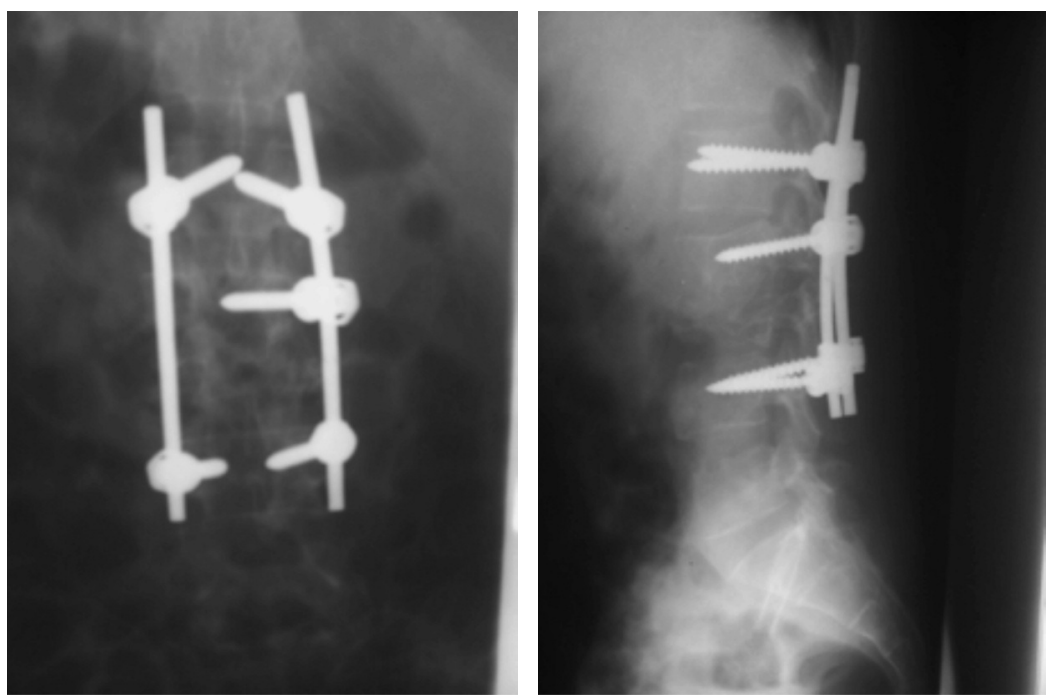

Fig. 3: Post-op. radiograph at 3 weeks (kyphotic angle 0/TLL angle 30). 

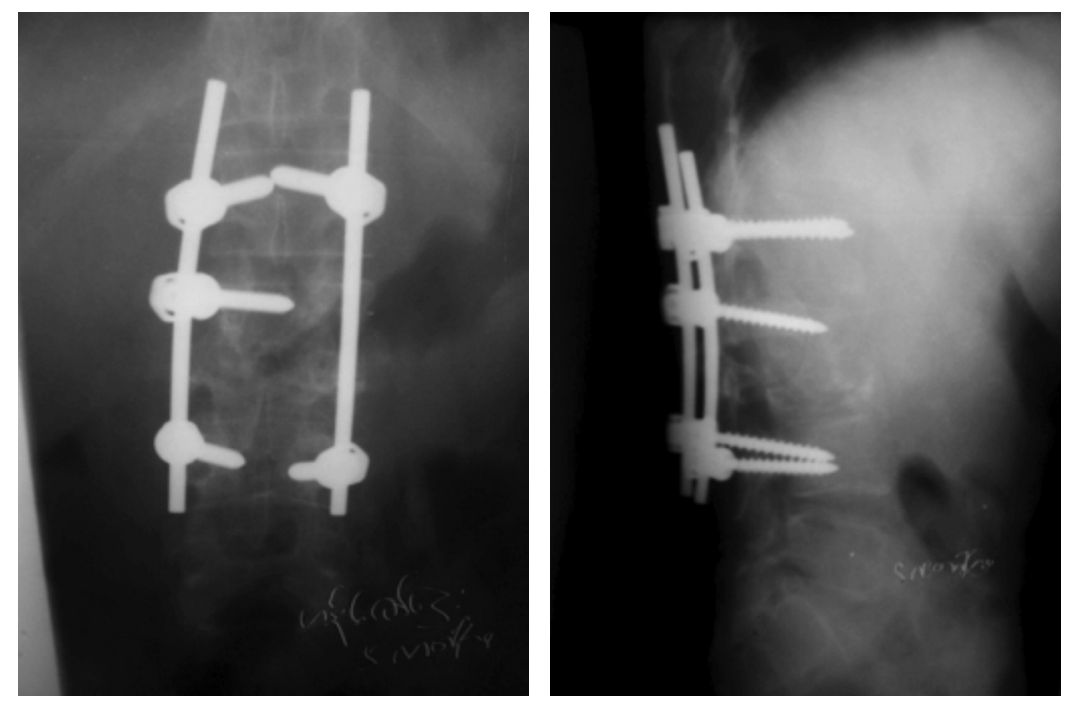

Fig. 4: Post-op radiograph showing correction at 3 months (kyphotic angle 0/TLL angle 30.
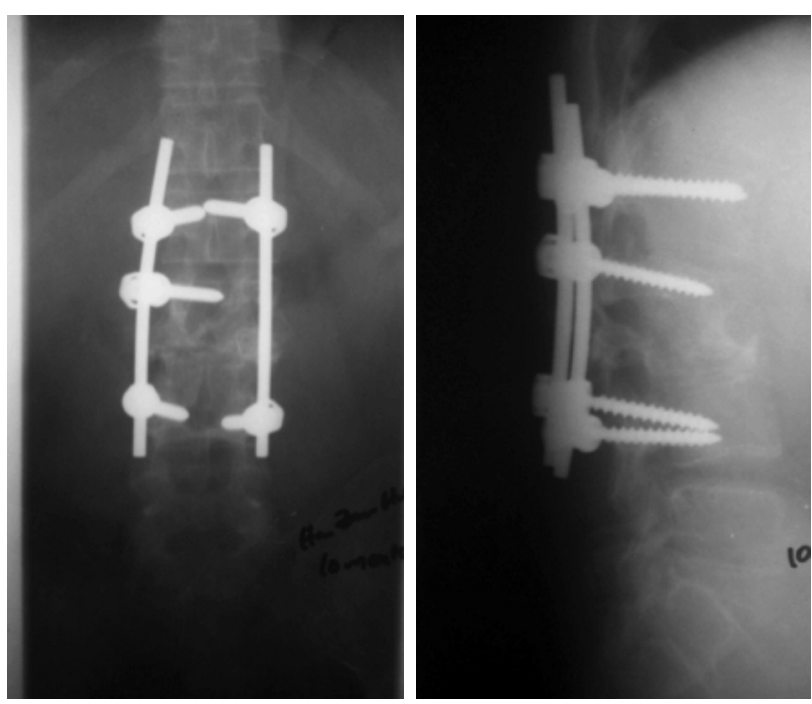

Fig. 5: Post-op. radiograph at 6 months (kyphotic angle 0/TLL angle 30).
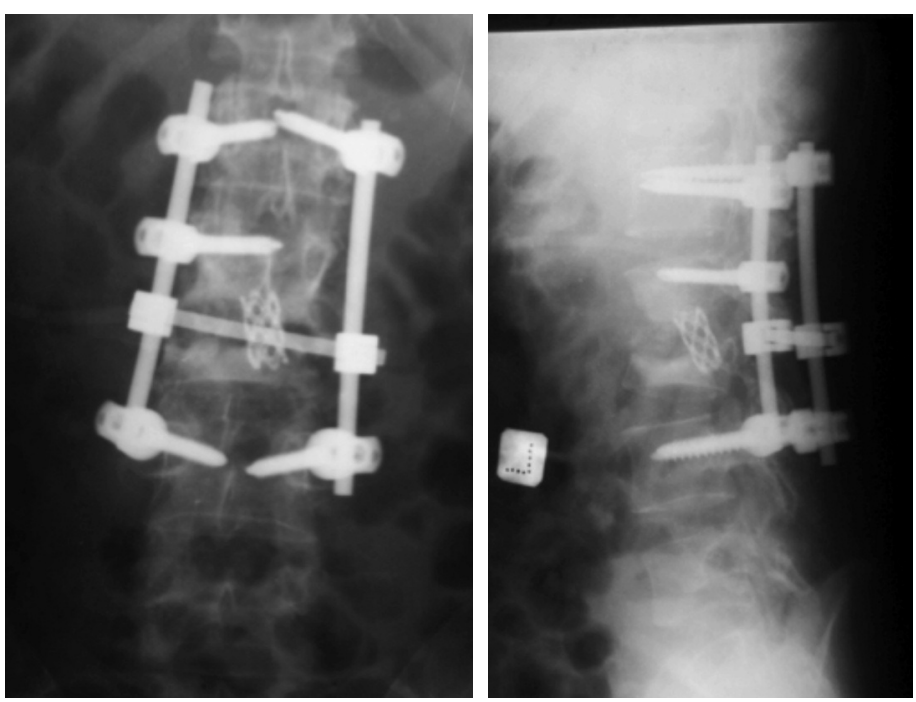

Fig. 7: Post-op radiograph at 3 weeks (kyphotic angle 0/TLL ang le 34).
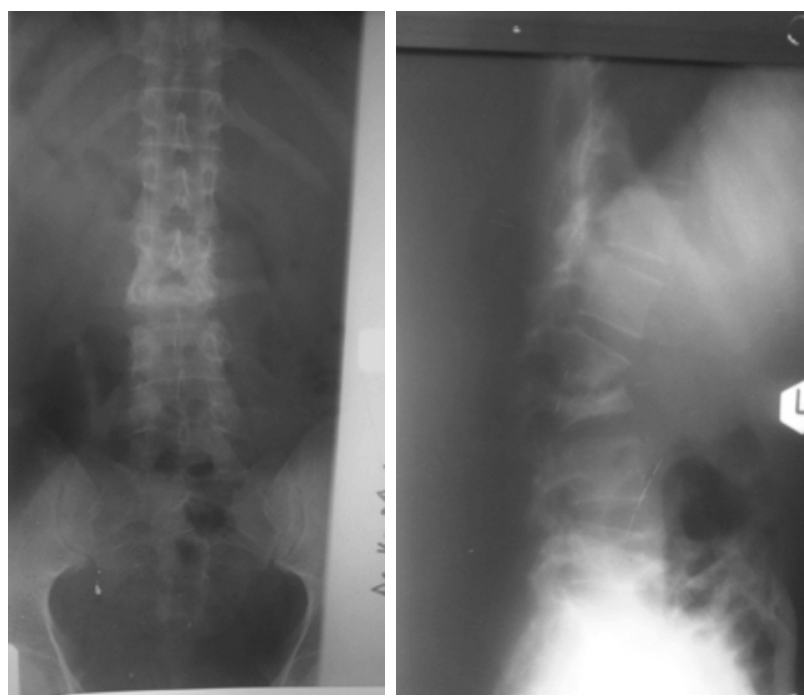

Fig. 6: Rediographs of tuberculous spine L2/3/ (kyphotic angle 40, TLL angle 0).

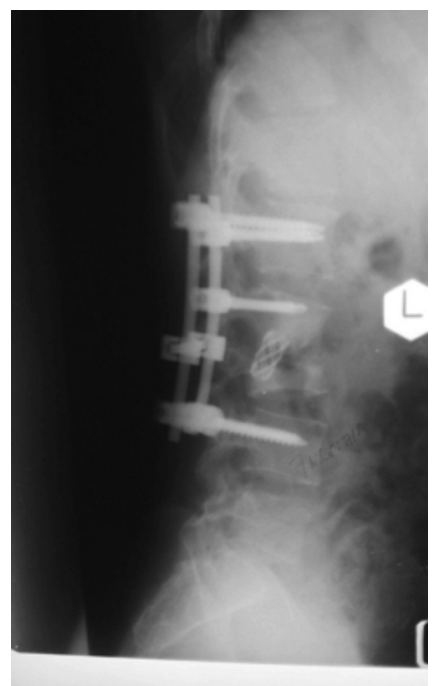

Fig. 8: Post op radiograph-at-;3 months (kyphotic angle 0/TLL angle 34). 


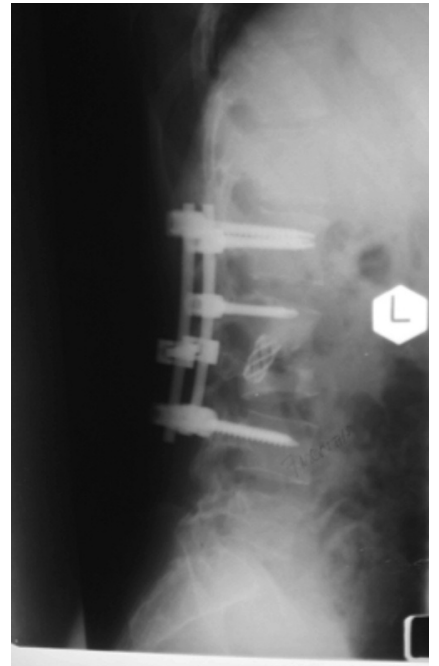

Fig. 9: Post-op radiograph -- at;6 months (kyphotic angle 0/TLL angle 34).

VAS OF BACK PAIN

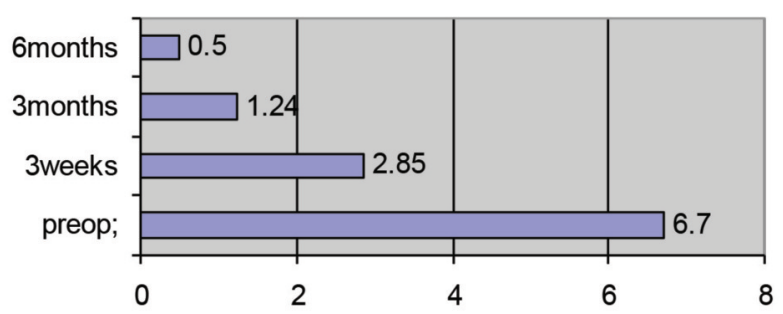

Fig. 11: Mean VAS back scores.

mean total lumbar lordotic angle

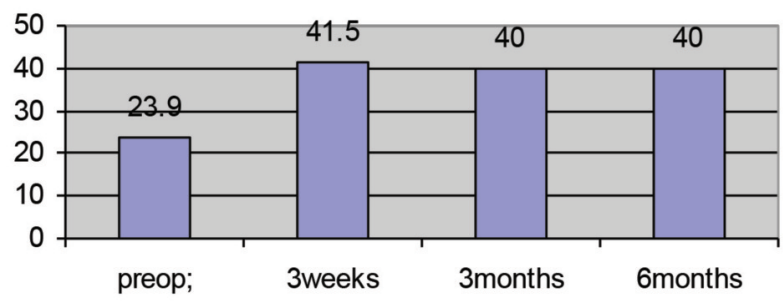

Fig. 13: Mean total lumbar lordosis angle.

disease if there is no marked abscess formation, spinal deformity and collapse or neurological deficit and vertebral instability ${ }^{20,21}$. Anterior debridement and arthrodesis allow access to the focal point of the disease directly, effective debridement of the focal point, rapid bony union with the grafts and prevent progressive collapse and kyphosis ${ }^{12,20,21}$.

However, it has been reported that such successful outcomes are not observed in progressive collapse and kyphosis,

\section{Modified MACNAB criteria}

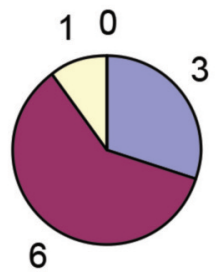

\begin{tabular}{|l|}
\hline$\square$ excellent \\
$\square$ good \\
$\square$ fair \\
$\square$ poor
\end{tabular}

Fig. 10: Modified MacNab Criteria at 6 months follow-up.

\section{Mean segmental kyphotic angle}

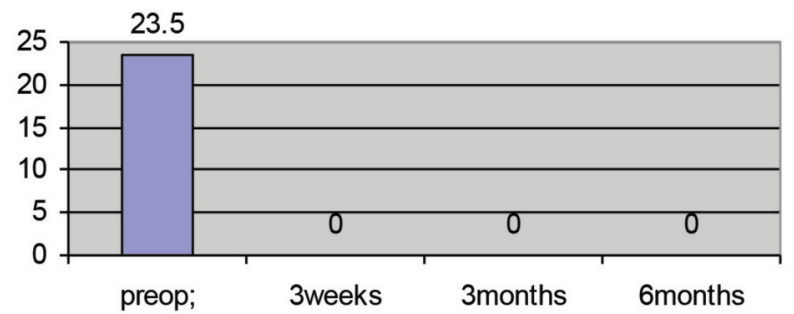

Fig. 12: Mean segmental kyphotic angle.

particularly in two or more levels of involvement, risks of

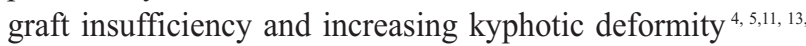
${ }^{17}$. Moon et al. stated that interbody fusion performed with classical anterior radical surgery per se is ineffective in the correction of kyphosis. Moon suggests that in addition to its failure in some cases to correct the existing kyphosis, anterior arthrodesis does not prevent the increase in kyphosis angle ${ }^{24}$. Rajasekaran et al. also reported progression in kyphosis particularly in anterior arthrodesis at two or more levels and recommended additional stabilization methods ${ }^{28}$.

Studies have shown that implants can be safely used in tuberculosis infections. In their study on stainless steel, Oga et al. reported that $\mathrm{M}$. tuberculosis has low adhesion capability and forms only a few microcolonies surrounded by a biofilm ${ }^{26}$. Benli et al., Y1lmaz et al. and Jin et al. showed that anterior implant can be safely used in tuberculous spondylitis ${ }^{8,16}$. In the literature, there are many studies showing that in addition to anterior debridement and arthrodesis, anterior or posterior stabilization is an effective method for correcting the existing kyphosis and maintaining the correction achieved ${ }^{6,8,10,14,17,25,26}$. 
It is clear that posterior instrumentation is more effective in kyphosis correction and prevention of late-term progression especially in kyphosis with two or more levels of involvement or in moderate or severe kyphosis ${ }^{14,15,17,26}$. In this study, mean VAS back scores significantly decreased at three months After six months mean VAS back scores decreased to 0.5 , because of satisfactory stabilization and clearance of infection. Mean segmental kyphotic angle was reduced from 23.5 to 0 degree after operations, and it was maintained up to 6 months. Effective correction of kyphosis is very important for long term mechanical stability and total sagittal balance, as correction of kyphosis shortens the lever arm and reduces the stress over the affected spine. In addition it maximizes the total contact surface area of adjacent vertebra which further promotes union and stability.

Mean total lumbar lordotic angle improved from 23.9 to 41.5, at three months mean value was 40 and remained so at six months. These sagittal plane angles were not much changed up to 6 months follow up but we need long term follow up to further comment, and to establish the status of fusion for all cases.
There were some weak points in this study, as we were unable to subject the results to statistical analysis because of the limited number of cases. We are collecting further cases to continue with this study. Even though modified MacNab criteria was used to evaluate, it only reflects patient satisfaction and some clinical results without scoring for functional outcome.

\section{CONCLUSION}

Radical debridement and reconstruction using posterior instrumentation is an acceptable surgical option for the treatment of lumbosacral spinal TB. It is clear that posterior instrumentation is more effective in kyphosis correction especially in kyphosis with two or more levels of involvement or in moderate or severe kyphosis, because it provides maximum contact surface to promote solid bony fusion, good clinical outcomes as well as improvement of mechanical function. The need for long term follow up is important to add further value to this study.

\section{REFERENCES}

1. Nene A, Bhojraj S. Results of nonsurgical treatment of thoracic spinal tuberculosis in adults. Spine J 2005; 5(1): 79-84.

2. Almeida A. Tuberculosis of the spine and spinal cord. Eur J Radiol 2005; 55: 193-201.

3. Benli IT, Alanay A, Akalin S, Kis M, Acaroglu E, Ates B, et al. Comparison of anterior instruments systems and the results of minimum 5 years follow up in the treatment of tuberculosis spondylitis. Kobe J Med Sci 2004; 50(5-6): 167-80.

4. Benli IT, Acaroglu E, Akalin S, Kis M, Duman E, Un A Anterior radical debridement and anterior instrumentation in tuberculosis spondylitis. Eur Spine J 2003; 12: 224-34.

5. Denis F, Armstrong GWD, Searls K, Matta L. Acute thoracolumbar burst fractures in the absence of neurologic deficit a comparison between operative and nonoperative treatment. Clin Orthop Relat Res. 1984; 189: 125-41.

6. Dvorak MF, Kwon BK, Fisher CG, Eiserloh HL 3rd, Boyd M,Wing PC Effectiveness of titanium mesh cylindrical cages in anterior column reconstruction after thoracic and lumbar vertebral body resection. Spine 2003; 28: 902-8.

7. Eck KR, Bridwell KH, Ungacta FF, Lapp MA, Lenke LG, Riew KD Analysis of titanium mesh cages in adults with minimum two-year follow-up. Spine 2000; 25: 2407-15.

8. Faraj AA. Anterior instrumentation for the treatment of spinal tuberculosis. JBJS (Am) 2001; 83: 463-4.

9. Frankel HL, Hancock DO, Hyskop G. The value of postural reduction in the initial management of closed injuries of the spine with paraplegia and tetraplegia. Paraplegia. 1069; 7: 179-92.

10. Fukuta S, Miyamoto K, Masuda T, Hosoe H, Kodama H, Nishimoto H, Sakaeda H, Shimizu K . Two-stage (posterior and anterior) surgical treatment using posterior spinal instrumentation for pyogenic and tuberculotic spondylitis. Spine 2003; 28: 302-8.

11. Hamzaog lu. A Granulomatous infection of the spine. Floman Y (ed) State of the art reviews, spinal infections. Hanley and Belfus, Philadelphia,1999; p. 45-78.

12. Hee HT, Majd ME, Holt RT, Pienkowski D.Better treatment of vertebral osteomyelitis using posterior stabilization and titanium mesh cages. J Spinal Disord Tech 2002; 15: 149-56.

13. Hilibrand AS, Quartararo LG, Moulton MRJ Spinal infections. In: Koval KJ (ed) Orthopaedic knowledge uptake 7. American Academy of Orthopaedic Surgeons, 2003; 661-72. 
14. Hodgson AR, Stock FE. Anterior spinal fusion: preliminary communication on radical treatment of Pott's disease and Pott's paraplegia. Clin Orthop Relat Res 1994 300: 16-23.

15. Janssens JP, de Haller. Spinal tuberculosis in a developed country. A review of 26 cases with special emphasis on abscesses and neurologic complications. Clin Orthop Relat Res 1990; 257: 67-75.

16. Jin D, Qu D, Chen J, Zhang H. One-stage anterior interbody autografting and instrumentation in primary surgical management of thoracolumbar spinal tuberculosis. Eur Spine J 2004; 13: 114-21.

17. Kim DJ, Yun YH, Moon SH, Riew KD. Posterior instrumentation using compressive laminar hooks and anterior interbody arthrodesis for the treatment of tuberculosis of the lower lumbar spine. Spine 2004; 29: 275-9.

18. Klockner C, Valencia R. Sagittal alignment after anterior debridement and fusion with or without additional posterior instrumentation in the treatment of pyogenic and tuberculous spondylodiscitis. Spine 2003; 15: 1036-42.

19. Laheri VJ, Badhe NP, Dewnany GT. Single stage decompression, anterior interbody fusion and posterior instrumentation for tuberculous kyphosis of the dorso-lumbar spine. Spinal Cord 2001; 39: 429-36.

20. Liljenqvist U, Lerner T, Bullmann V, Hackenberg L, Halm H, Winkelmann W. Titanium cages in the surgical treatment of severe vertebral osteomyelitis. Eur Spine J 2003; 12: 606-12.

21. Luk KD. Tuberculosis of the spine in the new millennium. Eur Spine J 1999; 8: 338-45.

22. Medical Research Council Working Party On Tuberculosis of the Spine .A 10-year assessments of controlled trials comparing debridement and anterior spinal fusion in the management of tuberculosis of the spine in patients on standard chemotherapy in Hong Kong. J Bone Jt Surg (Br) 1982; 64: 393-8.

22. Moon MS, Moon YW, Moon JL, Kim SS, Sun DH. Conservative treatment of tuberculosis of the lumbar and lumbosacral spine. Clin Orthop Relat Res 2002; 398: 40-9.

24. Moon MS, Woo YK, Lee KS, Ha KY, Kim SS, Sun DH. Posterior instrumentation and anterior interbody fusion for tuberculous kyphosis of dorsal and lumbar spine. Spine 1995; 20: 1910-16.

25. Moon MS pine update. Tuberculosis of the spine: controversies and new challenges. Spine 1997: 1791-7.

26. Oga M, Arizono T, Takasita M, Sugioka Y. Evaluation of the risk of instrumentation as a foreign body in spinal tuberculosis. Clinical and biologic study. Spine 1993; 18: 1890-4.

27. Ozdemir M, Us K, Ogun T The role of anterior spinal instrumentation and allograft fibula for treatment of Pott's disease. Spine 2003; 28: 474-9.

28. Rajasekaran S, Soundarapandian S: Progression of kyphosis in tuberculosis of the spine treated by anterior arthrodesis. Joint Bone Joint Surg Am 1989; 71: 1314-23. 